\title{
Inequalities for Discrete Möbius Groups in Infinite Dimension
}

\author{
Xi Fu \\ Department of Mathematics, Shaoxing University, Shaoxing, Zhejiang 312000, China \\ Correspondence should be addressed to Xi Fu; fuxi1000@yahoo.com.cn \\ Received 25 November 2012; Accepted 27 January 2013 \\ Academic Editor: Vladislav Kravchenko \\ Copyright (c) $2013 \mathrm{Xi} \mathrm{Fu}$. This is an open access article distributed under the Creative Commons Attribution License, which permits \\ unrestricted use, distribution, and reproduction in any medium, provided the original work is properly cited. \\ We establish some inequalities for discrete Möbius groups in infinite dimension, which are generalizations of the corresponding \\ results of Coa, 1996 and Gehring and Martin, 1991 in finite dimension.
}

\section{Introduction}

Let $\mathrm{GM}(n)$ denotes the group of all Möbius transformations in $\overline{\mathbb{R}}^{n}$ and $M(n)$ the subgroup of $\operatorname{GM}(n)$ consisting of all orientation-preserving Möbius transformations. The chordal distance between two points $x, y \in \overline{\mathbb{R}}^{n}$ is defined by (see [1])

$$
d(x, y)=|p(x)-p(y)|,
$$

where

$$
p(x)=e_{n+1}+\frac{2\left(x-e_{n+1}\right)}{\left|x-e_{n+1}\right|^{2}}
$$

is the stereographic projection from $\overline{\mathbb{R}}^{n}$ to the unit sphere $S^{n}$ in $\overline{\mathbb{R}}^{n+1}$. The chordal metric on $\operatorname{GM}(n)$ is defined by

$$
d(f, g)=\sup \left\{d(f(x), g(x)): x \in \overline{\mathbb{R}}^{n}\right\} .
$$

By using this metric and Clifford algebra, Gehring et al established a series of inequalities for discrete subgroups of $M(n)$. For example, Gehring and Martin [2] proved that if $\langle f, g\rangle$ is a discrete nonelementary subgroup of $M(2)$, then $\max \{d(f, I), d(g, I)\} \geq 2(\sqrt{2}-1)$; when $n>2$, by using Clifford algebra, Cao generalized it to $M(n)$ and obtained that if $\langle f, g\rangle$ is discrete nonelementary subgroup of $M(n)$ and $f$ is hyperbolic, then $\max \{d(f, I), d(g, I)\} \geq .683$ (see [3]); see [4] for the general cases. In 1991, Frunză [5] introduced the concept of Möbius transformations in infinite dimension and discussed the Clifford matrix representations of Möbius transformations in infinite dimension. Recently, by using the representations, $\mathrm{Li}$ [6-9] discussed the properties of Möbius transformations in infinite dimension and obtained several analogous Jørgensen's inequalities in $\operatorname{SL}(\Gamma)$. In this paper, we continue the study in this direction and some new inequalities for discrete subgroups of $\mathrm{SL}(\Gamma)$ are established. In Section 2, we give the preliminaries. The main results are given in Section 3.

\section{Preliminaries}

The Clifford algebra $\ell$ is the associative algebra over the real field $\mathbb{R}$, generated by a countable family $\left\{i_{k}\right\}_{k=1}^{\infty}$ subject to the following relations:

$$
i_{h} i_{k}=-i_{k} i_{h} \quad(h \neq k), \quad i_{k}^{2}=-1, \quad \forall h, k \geq 1
$$

and no others. Every element of $\ell$ can be expressed by the following type:

$$
a=\sum a_{I} I
$$

where $I=i_{v_{1}} i_{v_{2}} \cdots i_{v_{p}}, 1 \leq v_{1}<v_{2}<\cdots<v_{p}, p \leq n, n$ is a fixed natural number depending on $a, a_{I} \in \mathbb{R}$ are the coefficients, and $\sum_{I} a_{I}^{2}<\infty$. If $I=\emptyset$, then $a_{I}$ is called the real part of $a$ and denoted by $\operatorname{Re}(a)$; the remaining part is called the imaginary part of $a$ and denoted by $\operatorname{Im}(a)$. For each $a \in \ell$, the Euclidean norm is expressed by

$$
|a|=\sqrt{\sum_{I} a_{I}^{2}}=\sqrt{|\operatorname{Re}(a)|^{2}+|\operatorname{Im}(a)|^{2}} .
$$

The algebra $\ell$ has three important involutions. 
(1) “'”: replacing each $i_{k}(k \geq 1)$ of $a$ by $-i_{k}$, we get a new element $a^{\prime} . a \mapsto a^{\prime}$ is an isomorphism of $\ell$ :

$$
(a b)^{\prime}=a^{\prime} b^{\prime}, \quad(a+b)^{\prime}=a^{\prime}+b^{\prime},
$$

for $a, b \in \ell$.

(2) “*”: replacing each $i_{v_{1}} i_{v_{2}} \cdots i_{v_{p}}$ of $a$ by $i_{v_{p}} i_{v_{p-1}} \cdots i_{v_{1}}$. $a \mapsto a^{*}$ is an anti-isomorphism of $\ell$ :

$$
(a b)^{*}=b^{*} a^{*}, \quad(a+b)^{*}=b^{*}+a^{*} .
$$

(3) “-”: $\bar{a}=\left(a^{*}\right)^{\prime}=\left(a^{\prime}\right)^{*}$. It is obvious that $a \mapsto \bar{a}$ is also an anti-isomorphism of $\ell$.

For elements of the following type:

$$
x=x_{0}+x_{1} i_{1}+\cdots+x_{n} i_{n}+\cdots \in \ell
$$

we call them vectors. The set of all such vectors is denoted by $\ell_{2}$ and we let $\overline{\ell_{2}}=\ell_{2} \bigcup\{\infty\}$. For any $x \in \ell_{2}$, we have $x^{*}=x$ and $\bar{x}=x^{\prime}$. For $x, y \in \ell_{2}$, the inner product $(x \cdot y)$ of $x$ and $y$ is given by

$$
(x \cdot y)=x_{0} y_{0}+x_{1} y_{1}+\cdots+x_{n} y_{n}+\cdots,
$$

where $x=x_{0}+x_{1} i_{1}+\cdots+x_{n} i_{n}+\cdots, y=y_{0}+y_{1} i_{1}+\cdots+$ $y_{n} i_{n}+\cdots$.

It is easy to verify that any nonzero vector $x$ is invertible in $\ell$ with $x^{-1}=\bar{x} /|x|^{2}$. The inverse of a vector is invertible too. Since any product of nonzero vectors is invertible, we conclude that any product of nonzero vectors is invertible in $\ell$. The set of products of finitely many nonzero vectors is a multiplicative group, called Clifford group and denoted by $\Gamma$.

If a matrix $f=\left(\begin{array}{ll}a & b \\ c & d\end{array}\right)$ satisfies the following:

(1) $a, b, c, d \in \Gamma \bigcup\{0\}$;

(2) $a d^{*}-b c^{*}=1$;

(3) $a b^{*}, d^{*} b, c d^{*}, c^{*} a \in \ell_{2}$,

then we call $f$ a Clifford matrix in infinite dimension; the set of all such matrices is denoted by $\operatorname{SL}(\Gamma)$. The norm of $f$ is

$$
\|f\|=\sqrt{|a|^{2}+|b|^{2}+|c|^{2}+|d|^{2}} .
$$

Let

$$
f^{-1}=\left(\begin{array}{cc}
d^{*} & -b^{*} \\
-c^{*} & a^{*}
\end{array}\right) .
$$

Obviously, $f f^{-1}=f^{-1} f=I$; that is, $f^{-1}$ is the inverse of $f$. By a simple computation, we know that $\operatorname{SL}(\Gamma)$ is a multiplicative group of matrices.

For any $f= \pm\left(\begin{array}{ll}a & b \\ c & d\end{array}\right) \in \operatorname{SL}(\Gamma)$, the corresponding mapping

$$
f(x)=(a x+b)(c x+d)^{-1}
$$

is a bijection of $\overline{\ell_{2}}$ onto itself, which we call a Möbius transformation in infinite dimension. Correspondingly, the set of all such mappings is also a group, which is still denoted by $\operatorname{SL}(\Gamma)$.

Now, we give a classification to elements of $\operatorname{SL}(\Gamma)$ as follows. A nontrivial element $f \in \operatorname{SL}(\Gamma)$ is called:
(1) loxodromic if it is conjugate in $\operatorname{SL}(\Gamma)$ to $\left(\begin{array}{cc}r \lambda & 0 \\ 0 & r^{-1} \lambda^{\prime}\end{array}\right)$, where $r \in \mathbb{R} \backslash\{ \pm 1,0\}, \lambda \in \Gamma$, and $|\lambda|=1$; if $\lambda= \pm 1$, then $f$ is called hyperbolic;

(2) parabolic if it is conjugate in $\operatorname{SL}(\Gamma)$ to $\left(\begin{array}{ll}a & b \\ 0 & a^{\prime}\end{array}\right)$, where $a, b \in \Gamma,|a|=1, b \neq 0$, and $a b=b a^{\prime}$; if $a= \pm 1$, then $f$ is called strictly parabolic; if there is a positive integer $m$ such that $f^{m}$ is strictly parabolic and for any positive integer $m<k, f^{m}$ is not strictly, then $f$ is called $m$-strictly parabolic;

(3) elliptic if $f$ is neither loxodromic nor parabolic.

For a subgroup $G \subset \operatorname{SL}(\Gamma)$, we say that $G$ is discrete if and only if $f_{1}, f_{2}, \ldots \in G$ and $f_{i} \rightarrow I$ imply that $f_{i}=I$ for all sufficiently large $i$. Otherwise, $G$ is nondiscrete.

The following is a necessary condition for a two-generator discrete subgroup of $\mathrm{SL}(\Gamma)$ in which one generator is strictly parabolic.

Lemma 1 (see [9]). Suppose that $\langle f, g\rangle$ is a discrete subgroup of $S L(\Gamma)$ and $f, g$ are represented by

$$
f=\left(\begin{array}{ll}
1 & t \\
0 & 1
\end{array}\right), \quad g=\left(\begin{array}{ll}
a & b \\
c & d
\end{array}\right)
$$

where $c \neq 0$. Then

$$
|t c| \geq 1 \text {. }
$$

Let $x, y \in \overline{\ell_{2}}$, the chordal distance between them is defined as follows (see [10]):

$$
\begin{gathered}
d(x, y)=\frac{2|x-y|}{\sqrt{\left(1+|x|^{2}\right)\left(1+|y|^{2}\right)}}, \quad x, y \neq \infty, \\
d(x, y)=\frac{2}{\sqrt{\left(1+|x|^{2}\right)}}, \quad y=\infty .
\end{gathered}
$$

The chordal metric on $\operatorname{SL}(\Gamma)$ is set to be

$$
d(f, g)=\sup \left\{d(f(x), g(x)): x \in \overline{\ell_{2}}\right\} \text {. }
$$

We call $d(f)=d(f, I)$ the chordal norm of $f$. It is easy to see that $0 \leq d(f) \leq 2$. The readers can refer to $[5,6,8-10]$ for more details about Möbius groups in infinite dimension.

\section{The Main Results and Proofs}

Now we come to state and prove our main results.

Theorem 2. Suppose that $\langle f, g\rangle$ is a discrete subgroup of $S L(\Gamma)$ and $f, g$ are represented by

$$
f=\left(\begin{array}{cc}
r & 0 \\
0 & r^{-1}
\end{array}\right), \quad g=\left(\begin{array}{ll}
a & b \\
c & d
\end{array}\right),
$$


where $r \in \mathbb{R} \backslash\{ \pm 1,0\}$. If $g(\{0, \infty\}) \cap\{0, \infty\}=\emptyset$, then

$$
\begin{gathered}
d(f)^{2} \geq \frac{4\left(r-r^{-1}\right)^{2}}{\left(r+r^{-1}\right)^{2}}, \\
d(g)^{2} \geq \frac{4|b c|}{(2|b c|+1)}, \\
\left(r-r^{-1}\right)^{2}(|b c|+1) \geq 1 .
\end{gathered}
$$

Proof. Let $x=r^{-1}$. Then

$$
d^{2}(f(x), x) \geq \frac{4(|f(x)|-|x|)^{2}}{\left(1+|f(x)|^{2}\right)\left(1+|x|^{2}\right)}=\frac{4\left(r-r^{-1}\right)^{2}}{\left(r+r^{-1}\right)^{2}} .
$$

This proves (19). For (20), we let $x=0, y=\infty$. Since

$$
\begin{aligned}
& d(g)^{2} \geq d^{2}(g(x), x)=\frac{4\left|b d^{-1}\right|}{1+\left|b d^{-1}\right|^{2}}, \\
& d(g)^{2} \geq d^{2}(g(y), y)=\frac{4}{1+\left|a c^{-1}\right|^{2}},
\end{aligned}
$$

we have

$$
\begin{aligned}
& \frac{4-d(g)^{2}}{d(g)^{2}} \leq \frac{1}{\left|b d^{-1}\right|^{2}}, \\
& \frac{4-d(g)^{2}}{d(g)^{2}} \leq\left|a c^{-1}\right|^{2} .
\end{aligned}
$$

So (20) follows. For (21), we consider the Shimizu-Leutbecher sequence

$$
\begin{aligned}
& g_{0}=g, \quad g_{n+1}=g_{n} f g_{n}{ }^{-1}, \quad g_{n}=\left(\begin{array}{ll}
a_{n} & b_{n} \\
c_{n} & d_{n}
\end{array}\right), \\
& n=0,1, \ldots \text {. }
\end{aligned}
$$

Then

$$
\left(\begin{array}{ll}
a_{n+1} & b_{n+1} \\
c_{n+1} & d_{n+1}
\end{array}\right)=\left(\begin{array}{lll}
r a_{n} d_{n}^{*}-r^{-1} b_{n} c_{n}^{*} & -r a_{n} b_{n}^{*}+r^{-1} b_{n} a_{n}^{*} \\
r c_{n} d_{n}^{*}-r^{-1} d_{n} c_{n}^{*} & -r c_{n} d_{n}^{*}+r^{-1} d_{n} a_{n}^{*}
\end{array}\right) .
$$

It follows that

$$
\begin{aligned}
\left|b_{n+1} c_{n+1}\right| & =\left|a_{n} b_{n} c_{n} d_{n}\right|\left(r-r^{-1}\right)^{2} \\
& \leq\left|b_{n} c_{n}\right|\left(\left|b_{n} c_{n}\right|+1\right)\left(r-r^{-1}\right)^{2} .
\end{aligned}
$$

Suppose that $\mu=(1+|b c|)\left(r-r^{-1}\right)^{2}<1$. We obtain, by induction,

$$
\left|b_{n+1} c_{n+1}\right| \leq \mu^{n+1}|b c| .
$$

So,

$$
b_{n} c_{n} \longrightarrow 0, \quad a_{n} d_{n}^{*} \longrightarrow 1 .
$$

The above relation and (26) imply that

$$
a_{n} \longrightarrow r, \quad d_{n} \longrightarrow r^{-1} .
$$

Now

$$
\frac{\left|b_{n+1}\right|}{\left|b_{n}\right|}=\left|a_{n}\right|\left|r-r^{-1}\right| \longrightarrow\left|r\left(r-r^{-1}\right)\right|<r \sqrt{\mu} .
$$

Thus, by induction,

$$
\frac{b_{n}}{r^{n}} \longrightarrow 0, \quad c_{n} r^{n} \longrightarrow 0 .
$$

Since $\langle f, g\rangle$ is discrete and

$$
f^{-n} g_{2 n} f^{n}=\left(\begin{array}{cc}
a_{2 n} & r^{-2 n} b_{2 n} \\
r^{2 n} c_{2 n} & d_{2 n}
\end{array}\right),
$$

we have $b_{2 n}=c_{2 n}=0$ for sufficiently large $n$. Hence $g(\{0, \infty\}) \cap\{0, \infty\} \neq \emptyset$. It is a contradiction. Therefore, $(r-$ $\left.r^{-1}\right)^{2}(|b c|+1) \geq 1$.

By a simple caculation, we have the following.

Corollary 3. Suppose that $\langle f, g\rangle$ is a discrete subgroup of $\operatorname{SL}(2, \Gamma)$. If $f$ is hyperbolic with $f(x(f)=\{0, \infty\}$ and $g(\{0, \infty\}) \cap\{0, \infty\}=\emptyset$, then

$$
\max \{d(f), d(g)\} \geq \frac{\sqrt{6}}{3} .
$$

Theorem 4. Suppose that $\langle f, g\rangle$ is a discrete subgroup of $S L(\Gamma)$ and $f, g$ are represented by

$$
f=\left(\begin{array}{cc}
a & b \\
0 & a^{\prime}
\end{array}\right), \quad g=\left(\begin{array}{ll}
u & v \\
s & t
\end{array}\right) .
$$

If $f$ is $m$-strictly parabolic and $g(\infty) \neq \infty$, then

$$
\frac{\left(K^{6}+4 K^{4}\right)}{\left(4-K^{2}\right)^{2}} \geq \frac{2}{m^{2}},
$$

where $K=\max \{d(f), d(g)\}$.

Proof. Since $\langle f, g\rangle$ is discrete, by [9], we know that $|b s| \geq$ $1 / m$. It follows from

$$
\begin{gathered}
d^{2}(f(0), 0)=|b|^{2}\left(4-d^{2}(f(0), 0)\right), \\
4|s|^{2} \leq\|g\|^{2} d^{2}(g(\infty), \infty), \\
\|g\|^{2} \leq 2 \frac{4+d^{2}(g)}{4-d^{2}(g)},
\end{gathered}
$$

(cf. [11]), that

$$
\frac{d^{2}(f) d^{2}(g)\left(4+d^{2}(g)\right)}{\left(4-d^{2}(f)\right)\left(4-d^{2}(g)\right)} \geq \frac{2}{m^{2}} .
$$

So the inequality follows. 
Corollary 5. Suppose that $\langle f, g\rangle$ is discrete in $S L(\Gamma)$ and $f, g$ are represented by

$$
f=\left(\begin{array}{cc}
a & b \\
0 & a^{\prime}
\end{array}\right), \quad g=\left(\begin{array}{ll}
u & v \\
s & t
\end{array}\right),
$$

where $f$ is strictly parabolic. If $g(\infty) \neq \infty$, then

$$
\max \{d(f), d(g)\} \geq 1.22 \text {. }
$$

Remark 6. Following [6-8], we know that there are much differences between subgroups of $M(n)$ and SL( $\Gamma)$ such as the discreteness and the fixed points sets of elements, which play key roles in the proofs of [3, Theorem 3.11] and [2, Theorem 6.14]. This implies that our results cannot be deduced directly by the same methods used in $[2,3]$.

\section{Acknowledgment}

The research was partly supported by Tian-Yuan Foundation (no. 11226096).

\section{References}

[1] A. F. Beardon, The Geometry of Discrete Groups, vol. 91 of Graduate Texts in Mathematics, Springer, New York, NY, USA, 1983.

[2] F. W. Gehring and G. J. Martin, "Inequalities for Möbius transformations and discrete groups," Journal für die Reine und Angewandte Mathematik, vol. 418, pp. 31-76, 1991.

[3] C. Cao, "The chordal norm of discrete Möbius groups in several dimensions," Annales Academiae Scientiarum Fennicae Series A, vol. 21, no. 2, pp. 271-287, 1996.

[4] G. J. Martin, "On discrete Möbius groups in all dimensions: a generalization of Jørgensen's inequality," Acta Mathematica, vol. 163, no. 3-4, pp. 253-289, 1989.

[5] M. Frunză, "Möbius transformations in infinite dimension," Revue Roumaine de Mathématiques Pures et Appliquées, vol. 36, no. 7-8, pp. 369-376, 1991.

[6] L. Li, "Ball-preserving Möbius transformations in infinite dimension," Complex Variables and Elliptic Equations, vol. 54, no. 7, pp. 697-703, 2009.

[7] L. Li, “A generalization of Jørgensen's inequality to infinite dimension," New York Journal of Mathematics, vol. 17, pp. 41-49, 2011.

[8] L. Li, "Discreteness of Möbius groups in infinite dimension," Complex Variable, vol. 58, no. 1, pp. 109-112, 2013.

[9] L. L. Li, "An inequality on parabolic Möbius groups in infinite dimension and their application," Acta Mathematica Scientia Series A, vol. 32, no. 2, pp. 349-355, 2012.

[10] L. Li, "A class of Möbius transformations in infinite dimension," Journal of Jiangxi Normal University (Natural Sciences Edition), vol. 33, pp. 556-559, 2009.

[11] F. W. Gehring and G. J. Martin, "The matrix and chordal norms of Möbius transformations," in Complex Analysis, pp. 51-59, Birkhäuser, Basel, Switzerland, 1988. 


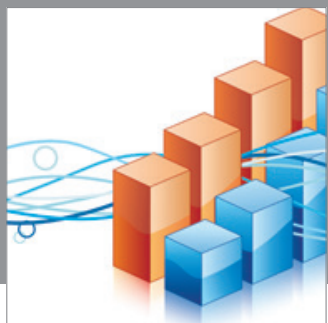

Advances in

Operations Research

mansans

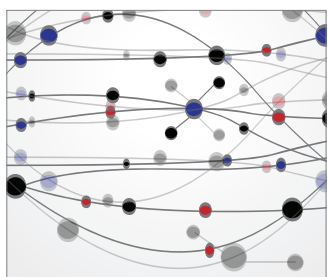

The Scientific World Journal
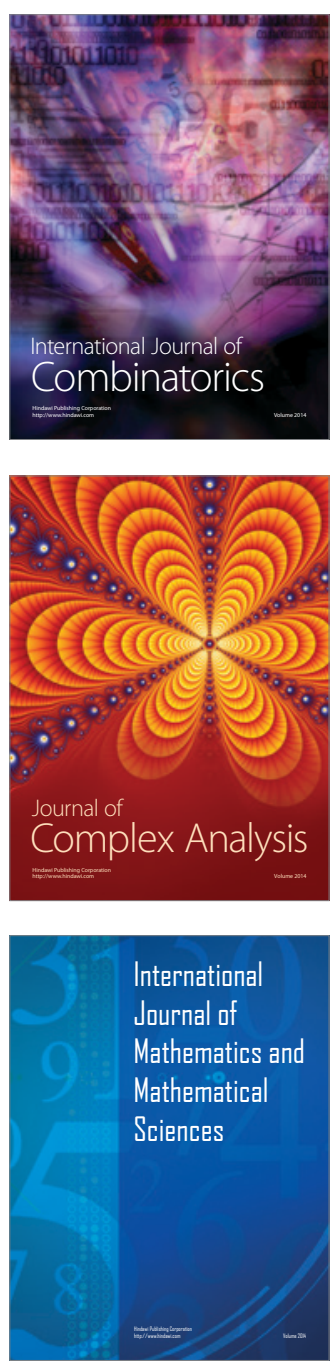
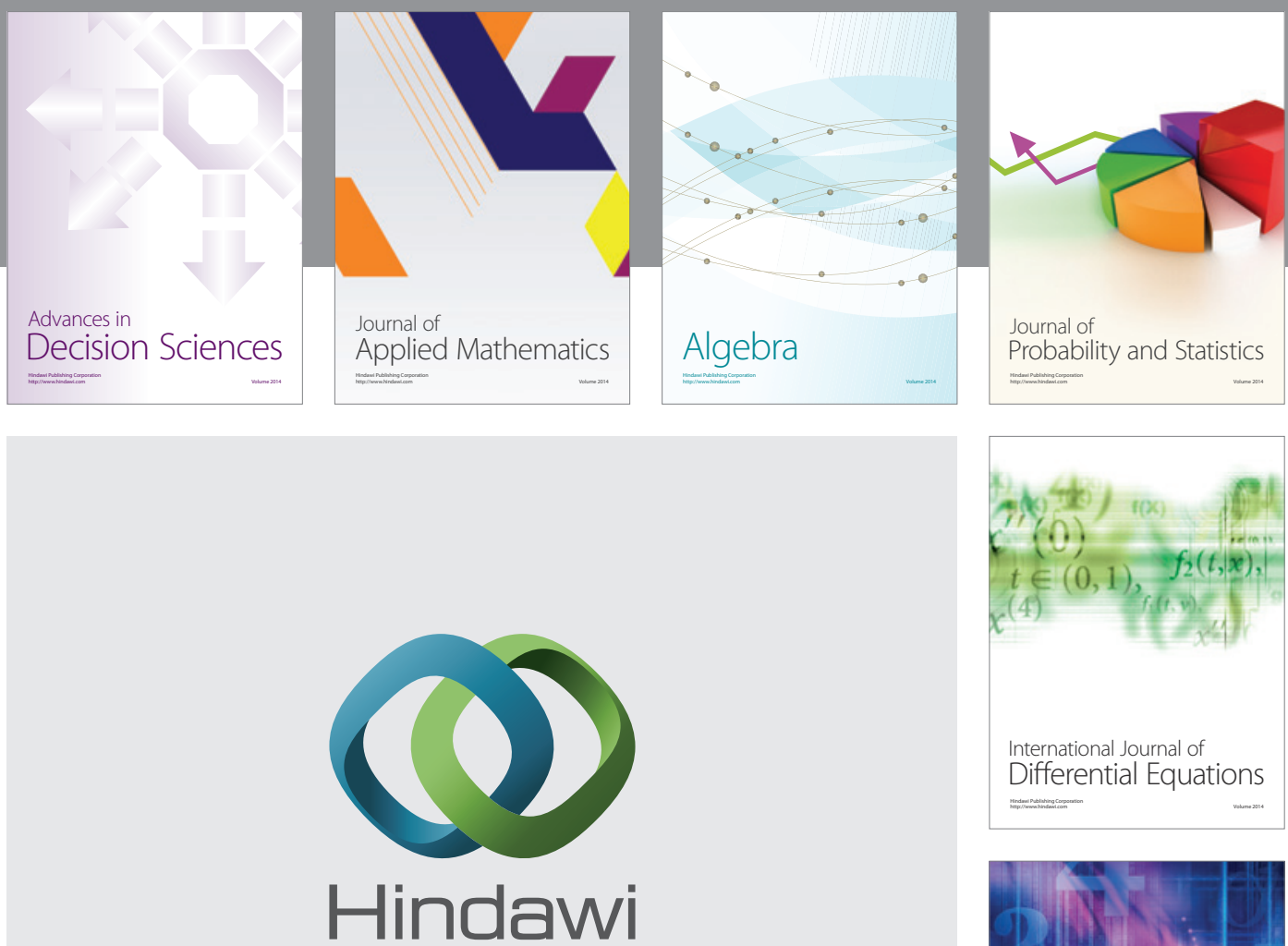

Submit your manuscripts at http://www.hindawi.com
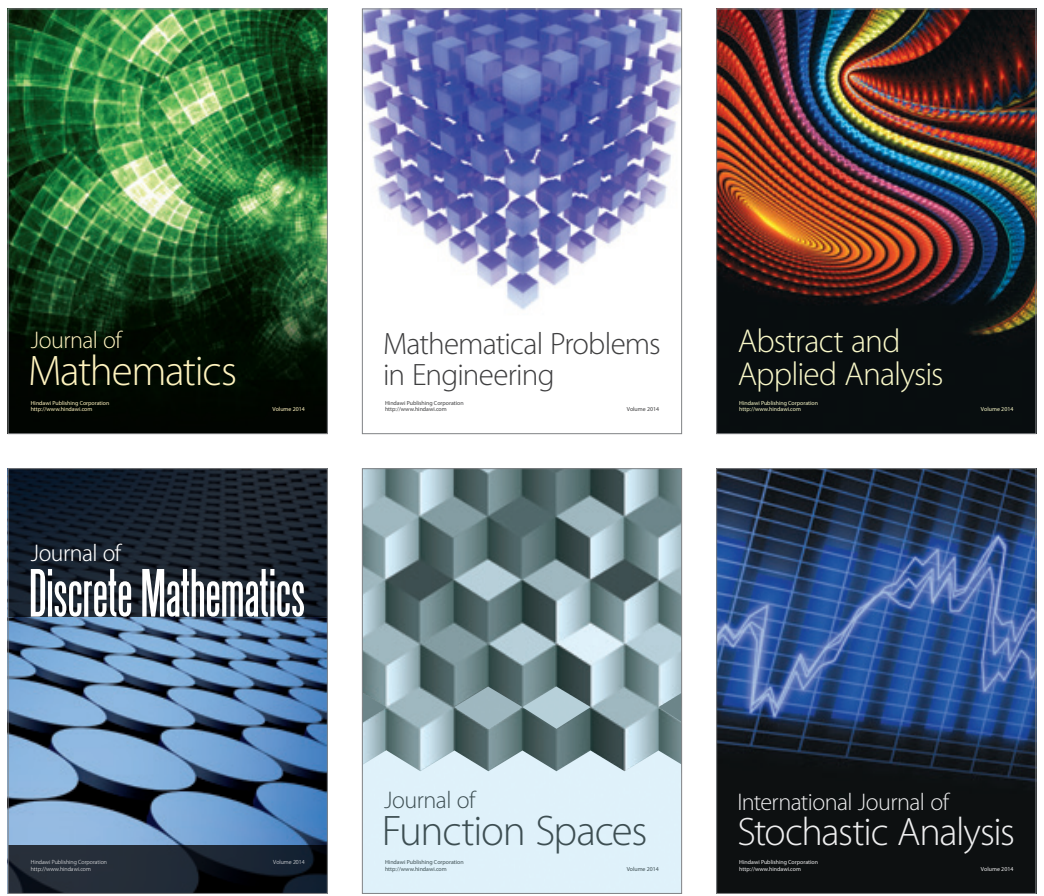

Journal of

Function Spaces

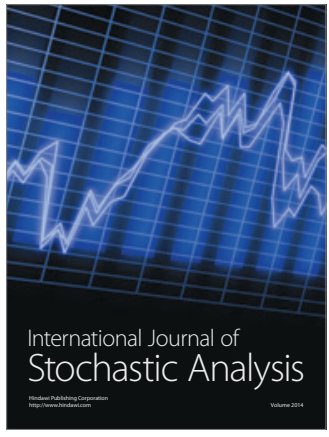

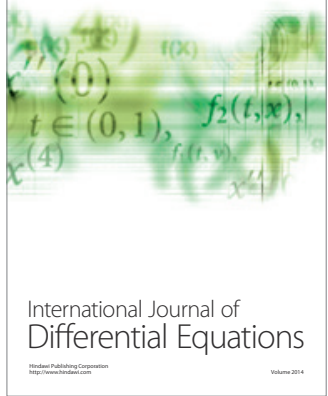
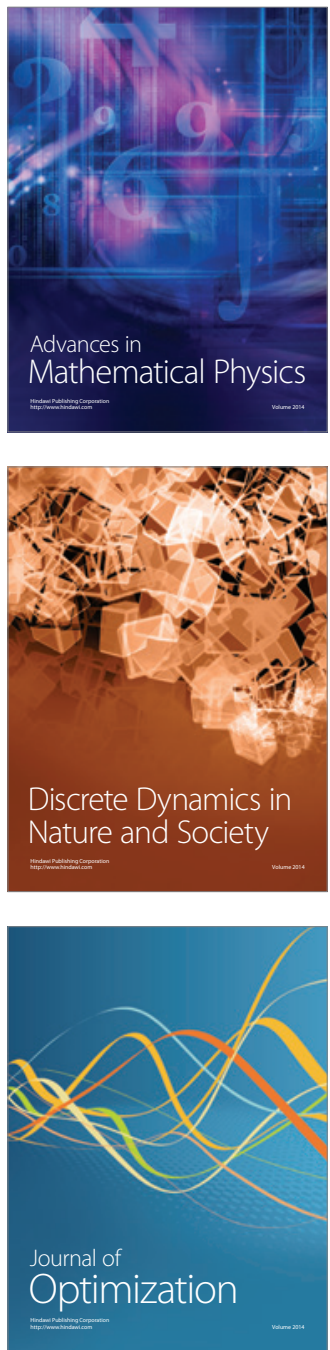